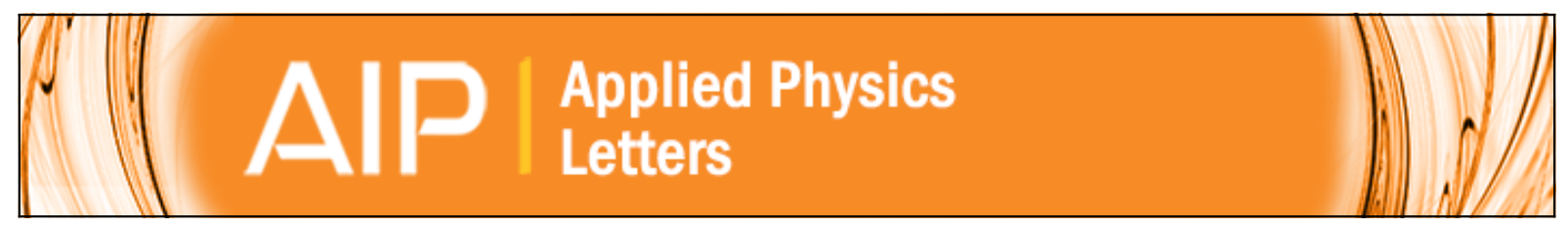

\title{
Impact of polydispersity on light propagation in colloidal photonic crystals
}

Mathieu Allard and Edward H. Sargent

Citation: Applied Physics Letters 85, 5887 (2004); doi: 10.1063/1.1835533

View online: http://dx.doi.org/10.1063/1.1835533

View Table of Contents: http://scitation.aip.org/content/aip/journal/apl/85/24?ver=pdfcov

Published by the AIP Publishing

Articles you may be interested in

Slab photonic crystals with dimer colloid bases

J. Appl. Phys. 115, 223514 (2014); 10.1063/1.4880743

Full spectrum enhancement of the light harvesting efficiency of dye sensitized solar cells by including colloidal photonic crystal multilayers

Appl. Phys. Lett. 88, 193110 (2006); 10.1063/1.2200746

Photon-directed colloidal crystallization

Appl. Phys. Lett. 85, 3760 (2004); 10.1063/1.1808471

Avoiding cracks in self-assembled photonic band-gap crystals

Appl. Phys. Lett. 84, 3573 (2004); 10.1063/1.1737066

Band spectroscopy of colloidal photonic crystal films

Appl. Phys. Lett. 84, 1239 (2004); 10.1063/1.1644913

\section{AIP $\left.\right|_{\text {APL Photonics }}$}

APL Photonics is pleased to announce Benjamin Eggleton as its Editor-in-Chief

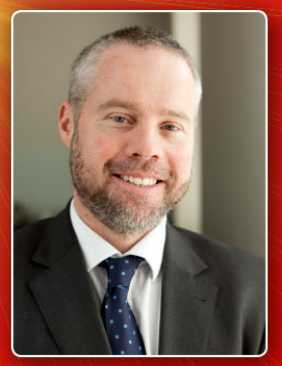




\title{
Impact of polydispersity on light propagation in colloidal photonic crystals
}

\author{
Mathieu Allard ${ }^{\text {a) }}$ and Edward H. Sargent \\ Department of Electrical and Computer Engineering, University of Toronto, Toronto, \\ Ontario, Canada M5S $3 G 4$
}

(Received 21 June 2004; accepted 19 October 2004)

\begin{abstract}
The influence of polydispersity, or variations in the size of particles, on the transmittance of slabs of colloidal photonic crystals is analyzed. A model based on simulated annealing simulates the assembly of particles with a given size distribution into a dense array. The model reveals the existence of a transition between ordered and random packing at a polydispersity between $3 \%$ and $4 \%$; this transition is associated with large changes in optical properties, in the form of greatly increased scattering and reduced contrast associated with the transmittance in and out of the stop band. The predictions of the model correspond closely to experimental measurements. (C) 2004 American Institute of Physics. [DOI: 10.1063/1.1835533]
\end{abstract}

Colloidal crystals offer a simple route to the fabrication of photonic crystals. ${ }^{1-3}$ The submicron-scale periodicity is ensured by self-assembly, without a need for external intervention or lithographic techniques. ${ }^{4-6}$ Many applications have been proposed for colloidal photonic crystals, such as optical filtering and switching. ${ }^{7,8}$

A certain amount of lattice disorder-defined as deviations from perfect periodicity-is, however, always present in self-assembled crystals. Disorder originates in the colloid polydispersity, that is, the size distribution among the particles, and also in stacking defects (dislocations, stacking faults, grain boundaries) in the crystal. By breaking the lattice periodicity, disorder prevents the free propagation of Bloch waves and causes incoherent scattering of light, ${ }^{9}$ which can lead to localization of light $;^{2}$ this competes with coherent Bragg scattering, on which many photonic applications of colloidal crystals rely. Before these applications can become a reality, the structural disorder in colloidal crystals and its impact on optical properties must be understood and quantified.

The incoherent scattering caused by lattice disorder has been characterized in light transmission experiments ${ }^{9-11}$ and also in coherent backscattering experiments, ${ }^{12,13}$ with which a photon mean free path can be measured. These studies have not, however, established a relation between the strength of the incoherent scattering and the underlying magnitude of the lattice disorder (expressed as the density of crystal defects or the colloid polydispersity). The impact of stacking faults on the transmission of light in otherwise perfectly ordered colloidal crystals has been assessed by numerical studies. ${ }^{14}$

$\mathrm{Li}$ and Zhang modeled the effect of polydispersity in colloidal crystals by introducing random size variations and displacements of the particles from their site in a face centered cubic lattice; they found that a polydispersity of $2 \%$ is sufficient to close the band gap in silicon-infiltrated crystals. ${ }^{15}$ In this model, particles were nonphysically allowed to overlap; additionally, the long-range perturbations to the lattice due to polydispersity were neglected. A superior approach to modeling the impact of polydispersity would be to simulate the actual formation of a densely packed array

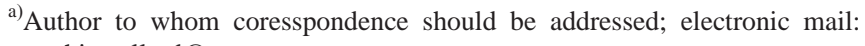
mathieu.allard@utoronto.ca from a collection of spheres with a given size distribution. Algorithms such as molecular dynamics ${ }^{16}$ or Monte Carlo simulations ${ }^{17}$ can be used to simulate the motion of collections of hard spheres. Though applicable to colloidal dispersions, these methods are better suited to study the thermodynamical properties of fluids of hard spheres than to generate fully settled arrays of particles.

In this letter, we introduce an algorithm that employs simulated annealing ${ }^{18}$ to produce physically realistic arrays of spheres with randomly distributed diameters. The algorithm works as follows. Initially, all particles are placed randomly in space above a single "seed" layer made of hexagonally packed particles. The energy of the system, $\mathcal{E}$, is defined as the sum of three contributions: (i) a onedimensional downward potential, (ii) a short-range interparticle attractive potential, and (iii) a hard-sphere repulsion potential, which prevents particles from overlapping. This energy function mimics the physical energy of a colloidal dispersion during the formation of colloidal crystals, ${ }^{6}$ where strong attractive capillary forces exist between neighboring particles and between particles and substrate. The algorithm searches for the lowest-energy arrangement by attempting to move each particle in turn by a small random displacement. In simulated annealing, while every move that decreases the energy is always accepted, one that increases it is accepted with probability $\exp (-\Delta \mathcal{E} / T)$, where $T$ is the "temperature" of the system. The initial temperature is set high enough that, at first, most moves are accepted; as time advances, the temperature is decreased exponentially. The nonzero but decreasing temperature in simulated annealing provides extra freedom to the system, and ensures that the final arrangement corresponds to the one with the lowest possible energy, or equivalently, the highest density. More details on the algorithm will be provided in a subsequent publication.

This algorithm was applied to collections of particles whose diameters followed a log-normal distribution with standard deviation $\sigma_{D}$. Several arrays were generated, with $\sigma_{D}$ ranging from $0 \%$ to $20 \%$; three of these are shown in Fig. 1 . The array generated from identical spheres $\left(\sigma_{D}=0\right)$ has a random hexagonal close-packed lattice (a mixture of face centered cubic and hexagonal close-packed lattices), which possesses the highest density possible for a lattice of hard spheres; other than unavoidable stacking faults, it contains no defects. The array generated with $\sigma_{D}=3 \%$ shows few 


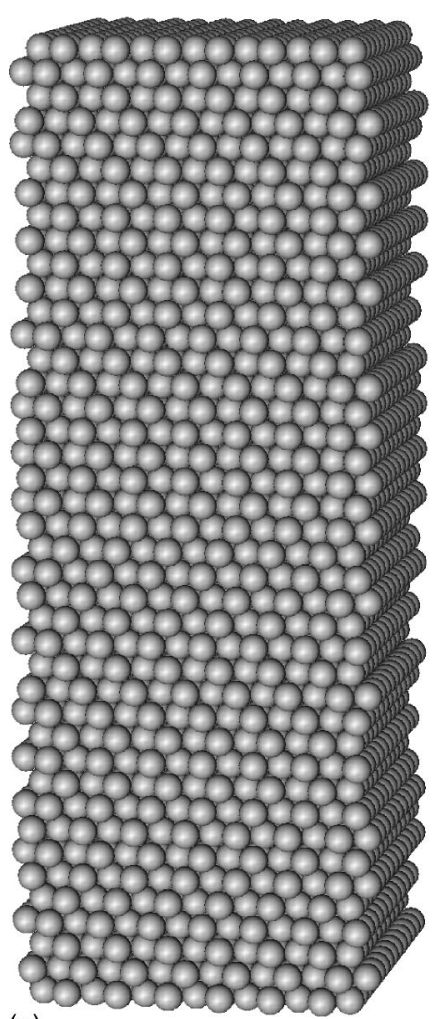

(a)

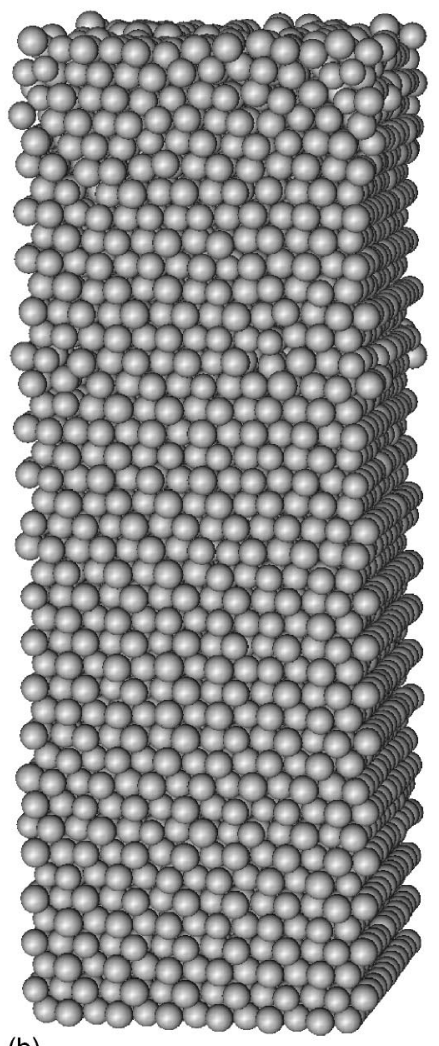

(b)

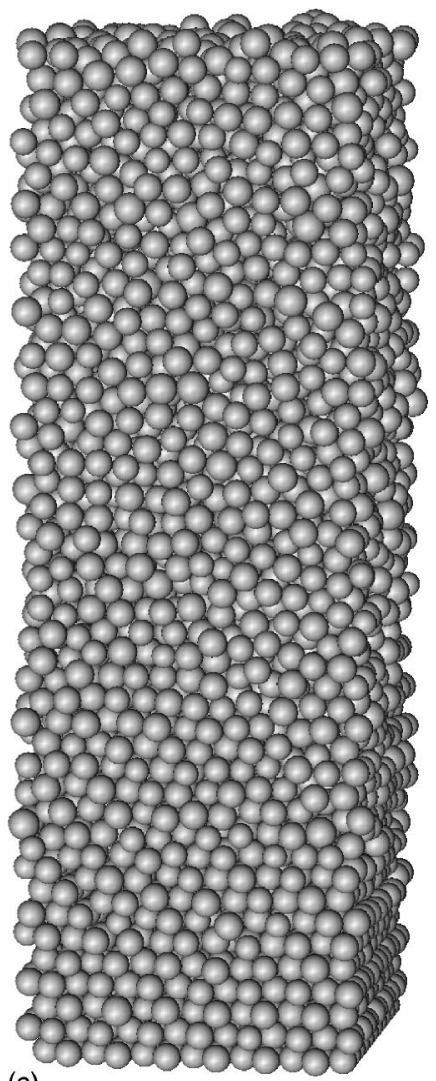

(c)

FIG. 1. Three-dimensional arrays of particles with different polydispersity $\left[\sigma_{D}\right]$ of (a) $0 \%$, (b) $3 \%$, and (c) $5 \%$. Each array has the same lateral dimensions and total number of particles as a close-packed array comprising $10 \times 12 \times 40$ particles. Periodic boundary conditions are used in both lateral directions. In the simulation, $10^{6}$ moves were attempted for every particle.

defects and the horizontal hexagonally packed layers are clearly visible. When $\sigma_{D}=5 \%$, however, the size variations among the particles are so large that an ordered (or crystalline) state is no longer favorable; the array then adopts a random-packed configuration, except in the few layers adjacent to the seed layer. Other simulations revealed that the transition from ordered to random packing occurred between $\sigma_{D}=3 \%$ and $4 \%$. Both the one-dimensional and the interparticle potential needed to be present to lead to dense arrays, although the results of the simulations were fairly insensitive to their relative magnitude.

The optical properties of the arrays were analyzed with the method of finite differences in time domain (FDTD). ${ }^{19}$ The combined time to generate an array and to run the FDTD computation was approximately two days on a personal computer. Figure 2 shows the transmittance and reflectance of each array, at normal incidence (on the top surface) and at frequencies in the vicinity of the first-order stop band; the total scattering, taken to be the intensity of all light not emerging from the crystal normally to the surfaces, is also plotted. When $\sigma_{D}=3 \%$, there is significant scattering outside the stop band, but, at frequencies inside the stop band, the reflectance is still close to unity, since most of the light is Bragg scattered before incoherent scattering can take place. When $\sigma_{D}=5 \%$, reflectance in the stop band reduces significantly, since the hexagonally packed planes that diffract light no longer exist in the random-packed array.

Figure 3 shows how optical properties change with increasing polydispersity. There is a sudden increase in the scattering at frequencies inside the stop band when $\sigma_{D}=4 \%$, corresponding to the transition from ordered to random pack- ing. At the same time, the contrast between the transmittance inside and outside the stop band decreases abruptly, and vanishes when $\sigma_{D}>5 \%$. On the same graph are also plotted similar results obtained from arrays generated using the

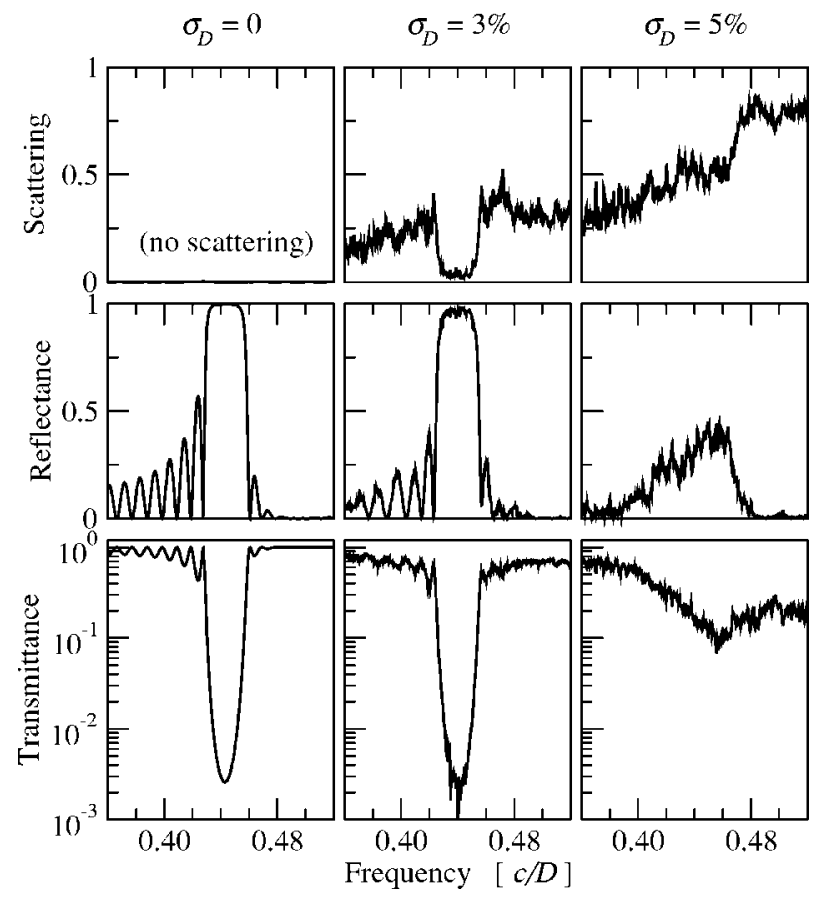

FIG. 2. Optical properties of the three arrays shown in Fig. 1, computed with FDTD; the particles have a refractive index of 1.5 and are embedded in air. 


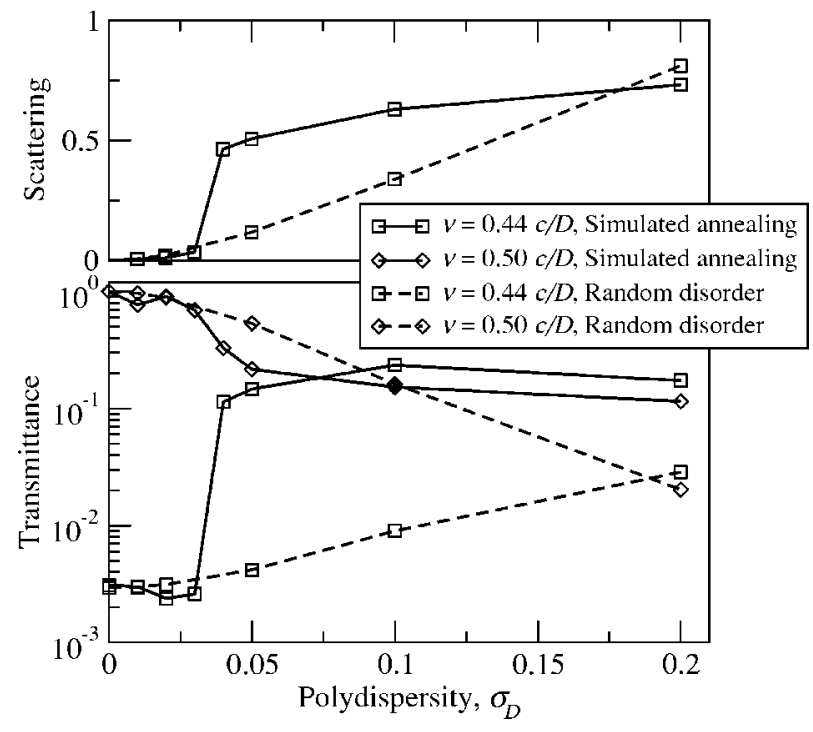

FIG. 3. Changes in transmittance and total scattering with polydispersity in arrays generated with the simulated-packing and the random-disorder model. Every array comprised 4800 particles and had the same lateral dimensions. The reflectance, transmittance and scattering were averaged over a spectral window of $0.01 \mathrm{c} / D$, either at the center of the stop band, at frequency $0.44 \mathrm{c} / D$, or outside the stop band, at frequency $0.50 \mathrm{c} / D$.

method detailed in Ref. 15, where each particle, in addition to having a randomly chosen diameter, is displaced by a random vector $\Delta \boldsymbol{r}$, with $\langle|\Delta \boldsymbol{r}|\rangle=\sigma_{D}$, from its position in a face centered cubic lattice. In this random-disorder model, the scattering increases much more slowly with increasing polydispersity; the contrast associated with the stop band in the transmittance spectrum also decreases more slowly. Thus, polydispersity has a much weaker effect in the random-disorder model.

To validate the modeling results, we compared them with experimental measurements of the transmittance of colloidal crystals grown using the capillarity method, ${ }^{6}$ using poly(methylmethacrylate) particles with diameter $630 \mathrm{~nm}$ and $n=1.49$. The thickness of the crystals was 84 layers, measured with a scanning electron microscope. Figure 4 shows the measured transmittance spectrum, along with the

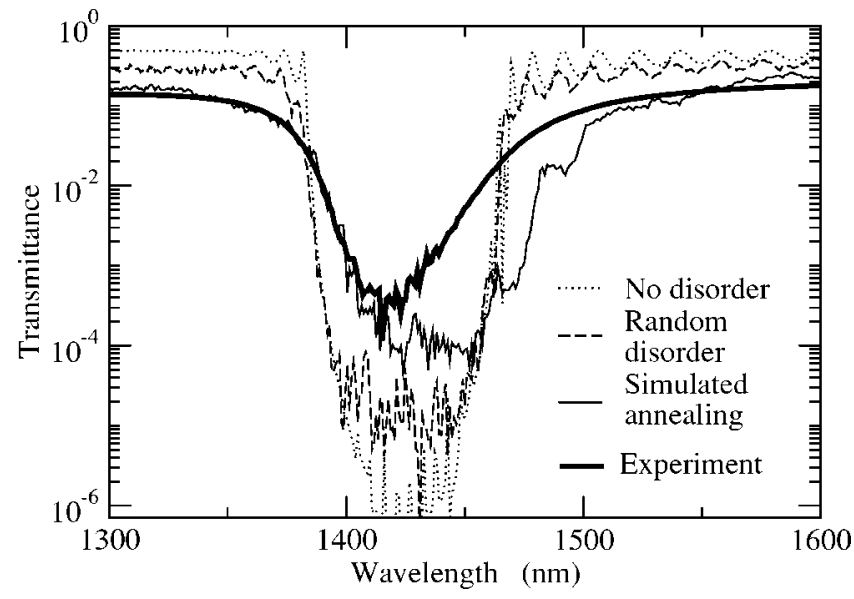

FIG. 4. Comparison of the transmittance measured on a colloidal crystal and the transmittance computed on a perfect face centered cubic array of spheres and on arrays generated with the random-disorder model and the simulatedpacking model, both with $\sigma_{D}=3 \%$. All arrays had a thickness of 84 layers. spectra computed numerically on three 84-layer arrays: one perfectly ordered, one generated with the random-disorder model and one generated with the simulated-annealing algorithm. The polydispersity used in the models was 3\%, corresponding to the value measured by dynamic light scattering in the colloidal dispersion. Only the spectrum predicted with the simulated-annealing model is in good agreement with the experimental result. The predicted and measured transmittance outside the stop band are both between 0.15 and 0.20 , while they are between $10^{-3}$ and $10^{-4}$ in the stop band. The width of the stop band is wider in the modeling spectrum; this is a numerical artefact caused by the finite lateral dimensions of the array (limited by available computer resources), which occasionally lead to a tilting of the normally horizontal planes of particles and to diffraction at different wavelengths.

The results presented here show that the randomdisorder model, though conceptually simple and convenient, fundamentally underestimates the effect of polydispersity and the lattice disorder that it produces. On the other hand, the simulated-packing model enables accurate predictions on the impact of polydispersity on the optical properties of colloidal crystals. Using this model, we found a transition from ordered to random packing when the polydispersity exceeded $3 \%$. This transition is associated with an abrupt degradation of the optical properties of the crystals, mostly in the form of increased scattering. Overall, these results point at the reduction of polydispersity as a very important factor in the improvement of the optical quality of colloidal crystals.

This work was supported by Nortel Networks, the Natural Sciences and Engineering Research Council of Canada through the Collaborative Research and Development Program, the Canada Foundation for Innovation, and the Canada Research Chairs Program.

${ }^{1}$ E. Yablonovitch, Phys. Rev. Lett. 58, 2059 (1987).

${ }^{2}$ S. John, Phys. Rev. Lett. 58, 2486 (1987).

${ }^{3}$ K. Busch and S. John, Phys. Rev. E 58, 3896 (1998).

${ }^{4}$ H. W. Deckman and J. H. Dunsmuir, Appl. Phys. Lett. 41, 377 (1982).

${ }^{5}$ H. Míguez, F. Meseguer, C. López, A. Blanco, J. S. Moya, J. Requena, A. Mifsud, and V. Fornés, Adv. Mater. (Weinheim, Ger.) 10, 480 (1998).

${ }^{6}$ P. Jiang, K. S. Hwang, and V. L. Colvin, Chem. Mater. 11, 2132 (1999).

${ }^{7}$ G. Pan, R. Kesavamoorthy, and S. Asher, Phys. Rev. Lett. 78, 3860 (1997).

${ }^{8}$ S. Park and Y. Xia, Langmuir 15, 266 (1999).

${ }^{9}$ V. N. Astratov, A. M. Adawi, S. Fricker, M. S. Skolnick, and D. M. Whittaker, Phys. Rev. B 66, 165215 (2002).

${ }^{10}$ Y. A. Vlasov, V. N. Astratov, A. V. Baryshev, A. A. Kaplyanskii, O. Z. Karimov, and M. F. Limonov, Phys. Rev. E 61, 5784 (2000).

${ }^{11}$ A. F. Koenderink and W. L. Vos, Phys. Rev. Lett. 91, 213902 (2003).

${ }^{12}$ A. F. Koenderink, M. Megens, G. van Soest, W. L. Vos, and A. Lagendjik, Phys. Lett. A 268, 104 (2000).

${ }^{13}$ J. Huang, N. Eradat, M. E. Raikh, Z. V. Vardeny, A. A. Zakhidov, and R. H. Baughman, Phys. Rev. Lett. 86, 4815 (2001).

${ }^{14}$ V. Yannopapas, A. Modinos, and N. Stefanou, Phys. Rev. B 68, 193205 (2003).

${ }^{15}$ Z.-Y. Li and Z.-Q. Zhang, Phys. Rev. B 62, 1516 (2000).

${ }^{16}$ D. C. Rapaport, The Art of Molecular Dynamics Simulation (Cambridge University Press, New York, 1995).

${ }^{17}$ M. E. J. Newman and G. T. Barkema, Monte Carlo Methods in Statistical Physics (Clarendon Press, Oxford, 1999).

${ }^{18}$ N. Metropolis, A. W. Rosenbluth, M. N. Rosenbluth, A. H. Teller, and E. Teller, J. Chem. Phys. 21, 1087 (1953).

${ }^{19}$ A. Taflove and S. C. Hagness, Computational Electrodynamics: The Finite-Difference Time-Domain Method, 2nd ed. (Artech, Boston, 2000). 